\section{Antigens by special delivery}

\section{By Tim Fulmer, Staff Writer}

DNA vaccines often require high and repeated dosing of antigen, which suggests suboptimal priming of the immune system and results in weak immunogenicity. ${ }^{1}$ Separate papers in Nature Biotechnology and the Journal of Clinical Investigation now show that directly targeting antigen to dendritic cells leads to a greater $T$ cell response and better DNA vaccine efficacy than nontargeted approaches. ${ }^{2,3}$

Because DNA vaccines can potentially incorporate multiple genes that encode a variety of immunostimulatory proteins, these therapeutics are theoretically ideal for targeting weakly immunogenic antigens associated with cancer and some persistent infections. But first-generation systemic vaccines have lacked efficacy.

The good news is that the role of dendritic cells (DCs) in initiating and controlling host cellular immune responses has been fleshed out. ${ }^{4,5}$ As a result, many of the newer vaccines in development have been designed to harness the immune-stimulating power of DCs to boost efficacy.

However, delivery of DNA by intradermal or intramuscular injection does not typically result in large numbers of DCs being activated to mature and migrate to the lymph nodes. Thus, companies in the space are exploring ways to improve direct delivery of DNA to DCs.

One approach has been to use autologous DCs loaded ex vivo with either protein or nucleotide antigens. A case in point is Dendreon Corp.'s Provenge sipuleucel-T, which consists of autologous DCs loaded with a fusion protein of prostatic acid phosphatase and an immunostimulatory cytokine. The vaccine is in a Phase III trial to treat hormone-refractory prostate cancer that the company hopes will satisfy efficacy requirements from an FDA approvable letter. ${ }^{6}$

The NBT and JCI papers offer a different approach-directly targeting and delivering antigen to DCs in vivo. The NBT paper suggests using a recombinant retroviral vector to home in on DCs, whereas the JCI paper proposes using a naked plasmid vector encoding both an antigen and a DC-targeting antibody.

In the NBT paper, researchers in David Baltimore's lab at the California Institute of Technology collaborated with Pin Wang's lab at the University of Southern California to design a lentiviral vector that encoded antigen and was enveloped by a Sindbis viral glycoprotein engineered to target the dendritic cell-specific intracellular adhesion molecule-3-grabbing nonintegrin protein (DC-SIGN protein) on the surface of DCs.

Baltimore is a professor of biology at Caltech. Wang, a corresponding author on the NBT paper, is a professor of chemical engineering and materials science at USC.
In the JCI paper, researchers at The Rockefeller University led by Ralph Steinman, professor and head of the laboratory of cellular physiology and immunology, designed a DNA plasmid vector that encoded a fusion protein comprised of antigen and single-chain antibody specific for the DEC205 receptor on DCs.

Both methods increased antigen-specific $\mathrm{CD}^{+}$and $\mathrm{CD}^{+} \mathrm{T}$ cell and antibody responses in vaccinated mice.

The data published in NBT show that mice receiving a single subcutaneous dose of the lentivector were completely protected from tumor challenge at 14 days postimmunization compared with controls.

In a separate experiment, mice that received lentivector 3 and 10 days following tumor challenge had reduced tumor growth and increased tumor regression on days 9-11, with no detectable tumor at the end of day 18 and thereafter. Unvaccinated mice showed progressive tumor growth.

Results in the JCI paper show that mice receiving a single, low-dose intramuscular injection of the DC-targeting plasmid were significantly protected from vaccinia-gag virus challenge at three months postimmunization compared with controls $(p<0.05)$.

According to Wang, "One of the advantages of the lentivector approach is that it relies on the natural antigen uptake process of dendritic cells." Furthermore, no co-stimulatory molecule is needed, he said.

The reason co-stimulation is not required remains unclear, but Baltimore expects one possibility is "the virus activates the dendritic cell, acting like a co-stimulatory molecule."

Wang told SciBX his group chose DC-SIGN in part because other researchers had shown that the C-type lectin DC-SIGN on DCs can function as an attachment receptor for the envelope proteins of several viruses, including Sindbis. ${ }^{7}$

Consequently, the Sindbis envelope glycoprotein suggested itself as a candidate for targeting and delivering lentivector to DCs. Moreover, Wang said, the glycoprotein could be engineered to bind exclusively to DC-SIGN and not to its other receptor, heparan sulfate, which is expressed by many cell types.

Baltimore added, "The lentivector's DC-targeting mechanism ensures safety through specificity. The Sindbis virus envelope protein is engineered to target only DC-SIGN, a receptor that's expressed by immature dendritic cells in peripheral tissue. Consequently, there is reduced risk of the vector infecting other host cells."

Wang said more studies are required on the response of regulatory $\mathrm{T}$ cells (Tregs) to the lentivector. "Tregs have a potentially suppressive effect on downstream killer $\mathrm{T}$ cells that are necessary for a robust immune response," he noted.

Steinman declined to comment on his team's research. He and his colleagues first characterized the involvement of the endocytic surface receptor DEC205 in DC antigen presentation more than a decade ago. ${ }^{8}$ Steinman has since developed a strategy to target vaccine antigens to the receptor on DCs in order to improve T cell response., ${ }^{9,10}$

David Spencer, CSO of Bellicum Pharmaceuticals Inc., wondered whether "exquisite DC targeting is really necessary, or will a less targeting approach still lead to robust response?"

He suggested the researchers on the NBT paper ought to investigate "whether the vaccine works better if one targets a different DC recep- 
tor, such as DEC205" and whether one could include a co-stimulatory adjuvant within the lentivector along with antigen.

Bellicum's genetically modified autologous DC-based cancer vaccine BP-GMAX-CD1 is expected to begin Phase I/II testing early this year to treat androgen-independent prostate cancer.

Charles Nicolette, CSO and VP of R\&D at Argos Therapeutics Inc., told SciBX, "The NBT paper offers a very elegant approach to potentially designing vaccines that target dendritic cells in vivo. However, a number of issues still must be addressed before the vaccine might find its way into the clinic."

For example, said Nicolette, "there's the concern that in diseases where dendritic cell function is compromised, such as herpes simplex or cytomegalovirus, targeting these cells may not result in successful immunization."

Argos' ex vivo RNA-loaded autologous DC vaccines AGS-003, AGS-004 and AGS-005 are in Phase II testing to treat metastatic renal cell carcinoma, HIV and chronic lymphocytic cell leukemia, respectively.

A general concern with any retrovirus-based vaccine, Nicolette added, "is the high regulatory hurdles that are associated with such approaches. In particular, there is the safety risk that when the lentivirus inserts into the host chromosome it could, for example, activate an oncogene."

Nicolette said he would like to see the lentivector studied in "more challenging" preclinical disease models.

"The chicken ovalbumin tumor antigen used in the paper is highly immunogenic," he said. "For the design of potential cancer vaccines, it would be more relevant to look at preclinical models characterized by weakly immunogenic, nonmutated self-antigens, as often occur in cancer. One such model is the B16 murine melanoma model."

Spencer agreed that the next steps should include evaluating the lentivector-based vaccine in "physiologically relevant, less immunogenic models."

Wang told SciBX that future research will look at additional antigens in both infectious disease and cancer.

"It's impressive that the researchers saw a therapeutic effect after a single administration of viral vector," said Michael Fons, VP of corporate development at Inovio Biomedical Corp. "This suggests that viral targeting of dendritic cells could avoid the requirement of repeat dosing that's often seen with other DNA vaccine delivery strategies."

Fons added that "a study in mice cannot rule out the possibility that repeat dosing will be required in larger animals and humans-which, over time, could cause patients to mount an immune response to vaccine components and, ultimately, leave patients refractory to further treatment."

Inovio has several ongoing Phase I/II trials of DNA vaccines to treat cancer and HCV infection. The company uses electroporation to facilitate uptake of naked DNA plasmid by tumor, muscle, skin or mucosal tissue. The company's approach does not specifically target DCs.

The most advanced in vivo DC-targeting vaccine is CDX-1307 from Celldex Therapeutics Inc. The fusion protein consists of an antibody that targets DC-surface mannose receptors and a whole protein tumor antigen, human chorionic gonadotropin hormone $\beta$-chain, which is overexpressed in multiple cancers.

The vaccine was safe and well tolerated in a Phase I trial in patients with incurable colorectal, pancreatic and bladder cancers, said Venky Ramakrishna, assistant director of immunology at Celldex. A Phase IIa/ IIb trial in those cancers is ongoing.
A key challenge with in vivo DC-targeting strategies, said Ramakrishna, is that in humans, "although subdermal vaccination may activate local dendritic cells, it may not at all result in their migration to the proper lymph nodes." Consequently, he said, companies that continue to develop in vivo DC-targeting approaches, including Celldex, will probably turn to co-stimulatory adjuvants that enhance DC activation and migration.

Added Ramakrishna, "While direct vaccination into specific lymph nodes might avoid the need for such adjuvants, it is a highly invasive procedure for which patient consent is difficult to procure and therefore probably useful only as a last resort."

The most advanced in vivo lentivector-based therapy is Oxford BioMedica plc's ProSavin, which is in a Phase I/II trial to treat Parkinson's disease. ProSavin consists of a lentivector encoding three enzymes required for dopamine production and a vesicular stomatitis virus envelope glycoprotein. The construct is delivered directly into the brain's striatum.

Stuart Naylor, the company's SVP of R\&D, told SciBX that "engineering the lentiviral vector to specifically target dendritic cells is an impressive feat" for the NBT authors. "However, this will also likely increase the complexity of the manufacturing process. And in fact, challenges associated with the consistent manufacture of lentiviral constructs are typically the main bottleneck into the clinic."

Naylor said there are other ways to design in vivo DC-targeted lentivector vaccines that are potentially less complex than Baltimore and Wang's approach. For example, he said, Mary Collins and colleagues at University College London have restricted lentivector antigen gene expression to DCs by using a DC-specific promoter rather than engineering DC-specificity into a viral envelope protein.

Earlier this year, Collins and colleagues published preclinical proof-ofconcept data demonstrating their strategy in mice. ${ }^{11}$

\section{REFERENCES}

1. Rice, J. et al. Nat. Rev. Cancer 8, 108-120 (2008)

2. Yang, L. et al. Nat. Biotechnol.; published online Feb. 24, 2008; doi:10.1038/nbt1390

Contact: Pin Wang, University of Southern California, Los Angeles, Calif. e-mail: pinwang@usc.edu

Contact: David Baltimore, California Institute of Technology, Pasadena, Calif. e-mail: baltimo@caltech.edu

3. Nchinda, G. et al. J. Clin. Invest.; published online March 6, 2007; doi:10.1172/JCl34224

Contact: Ralph M. Steinman, The Rockefeller University, New York, N.Y. e-mail: steinma@mail.rockefeller.edu

4. Figdor, C. et al. Nat. Med. 10, 475-480 (2004)

5. Tacken, P. et al. Nat. Rev. Immunol. 7, 790-802 (2007)

6. Flanagan, M. BioCentury 15(22), A10-A12; May 14, 2007

7. Klimstra, W.B. et al. J. Virol. 77, 12022-12032 (2003)

8. Jiang, W. et al. Nature 375, 151-155 (1995)

9. Bonifaz, L.C. et al. J. Exp. Med. 199, 815-824 (2004)

10. Bozzacco, L. et al. Proc. Natl. Acad. Sci. USA 104, 1289-1294 (2007)

11. Lopes, L. et al. J. Virol. 82, 86-95 (2008)

COMPANIES AND INSTITUTIONS MENTIONED

Argos Therapeutics Inc., Durham, N.C.

Bellicum Pharmaceuticals Inc., Houston, Texas

California Institute of Technology, Pasadena, Calif.

Celldex Therapeutics Inc., Phillipsburg, N.J.

Dendreon Corp. (NASDAQ:DNDN), Seattle, Wash.

Inovio Biomedical Corp. (AMEX:INO), San Diego, Calif.

Oxford BioMedica plc (LSE:OXB), Oxford, U.K.

The Rockefeller University, New York, N.Y.

University College London, London, U.K.

University of Southern California, Los Angeles, Calif. 